\title{
BNIPL-2 promotes the invasion and metastasis of human hepatocellular carcinoma cells
}

\author{
LI XIE ${ }^{1,2}$, WENXIN QIN ${ }^{1}$, JINJUN LI $^{1}$, XIANGHUO HE ${ }^{1}$, HAITAO ZHANG $^{3}$, GENFU YAO $^{1}$, \\ HUIQUN SHU ${ }^{1}$, MING YAO $^{1}$, DAFANG WAN ${ }^{1}$ and JIANREN GU ${ }^{1}$ \\ ${ }^{1}$ National Laboratory for Oncogenes and Related Genes, WHO Collaborating Center for Research on Cancer, Shanghai \\ Cancer Institute, Shanghai Jiao Tong University, Shanghai 200032; ${ }^{2}$ Department of Oncology, Shandong \\ Cancer Hospital and Cancer Institute, Jinan 250017; ${ }^{3}$ School of Environmental and Biological \\ Science and Technology, Dalian University of Technology, Dalian 116023, P.R. China
}

Received November 1, 2006; Accepted December 4, 2006

\begin{abstract}
Enhanced cell migration and invasion play key roles in cancer metastasis. However, the molecules involved in this process are not fully understood. In this study, a fulllength human BNIPL-2 (Bcl-2/adenovirus E1B $19 \mathrm{kDa}$ interacting protein 2 like-2) cDNA was transfected into human hepatocellular carcinoma cells with low metastatic potential (MHCC97-L). The in vitro and in vivo effects of BNIPL-2 on cell invasion and metastasis were examined. In vitro analysis showed that the overexpression of BNIPL-2 increases cell invasion and promotes cell migration. The rates of intrahepatic and pulmonary metastasis in nude mice were also increased. Cdc42 activation assays and immunoblot analysis indicated that the activation of $\mathrm{Cdc} 42$ and the upregulation of CD44 were involved in the metastasis of cancer cells. The overexpression of BNIPL-2 promotes the invasion and metastasis of MHCC97-L cells. Thus, BNIPL-2 is a gene related with cancer metastasis.
\end{abstract}

\section{Introduction}

Tumor invasion and metastasis are major obstacles for successful cancer therapy (1). Cancer metastasis is a multistep process that involves the dissociation of tumor cells from the primary tumor followed by their entry into the circulation, extravasation from the vascular system and proliferation into tumor masses at secondary tissue sites. Failure at any of these steps can block the entire metastatic

Correspondence to: Dr Wenxin Qin and Dr Dafang Wan, National Laboratory for Oncogenes and Related Genes, WHO Collaborating Center for Research on Cancer, Shanghai Cancer Institute, Shanghai Jiao Tong University, 25/Ln 2200 Xie-Tu Road, Shanghai 200032, P.R. China

E-mail: qinwenxin@smmail.cn

Key words: BNIPL-2, invasion, cancer metastasis process $(2,3)$. Determining which proteins are required for cancer metastasis is an important first step in developing therapeutics aimed at limiting the metastasis or dispersal of tumor cells.

Important biological processes in the development of metastasis include the increased migration and invasion of tumor cells. Cell migration is associated with the reorganization of the actin cytoskeleton that triggers changes in cell morphology. The activation of members of the Rho family of GTPases, such as RhoA, Rac1, and Cdc42, controls the dynamics of the actin cytoskeleton. Thus, they represent key regulatory molecules during cell migration (4). BNIPL-2 was identified as a novel member of the BNIPL family by our laboratory and we found that it interacted with Cdc42GAP and suppressed the GAP activity of Cdc42GAP towards $\mathrm{Cdc} 42$ (5). According to previous results, the $\mathrm{BCH}$ domain (BNIP-2 and Cdc42GAP homology) induces short and long pseudopodia, and is subsequently needed to trigger cell migration (6). As BNIPL-2 has a $\mathrm{BCH}$ domain, it could be involved in cell migration and cancer metastasis.

We report here that the overexpression of BNIPL-2 increased cell migration and invasion in vitro and promoted the metastasis of hepatocellular carcinoma (HCC) cells in vivo. Furthermore, BNIPL-2 promotes the activition of small GTPases such as $\mathrm{Cdc} 42$, resulting in the formation of filopodia. Our data show the molecular mechanisms mediating the BNIPL-2-induced migration and metastasis of cancer cells.

\section{Materials and methods}

Cells and cell culture. Human hepatocellular carcinoma cells with low metastatic potential (MHCC97-L) were obtained from the Liver Cancer Institute, Zhongshan Hospital, Fudan University (7). The cells were cultured in DMEM medium (Gibco BRL-Life Technologies, Grand Island, NY, USA), containing $10 \%$ quality fetal bovine serum (Hyclone, Logan, UT, USA), 100 units $/ \mathrm{ml}$ penicillin, and $100 \mu \mathrm{g} / \mathrm{ml}$ streptomycin at $37^{\circ} \mathrm{C}$ in $5 \% \mathrm{CO}_{2}$.

Transfection. A full-length human BNIPL-2 cDNA was cloned into the pEGFP-N1 plasmid (Clontech Laboratories, 
Inc., Palo Alto, CA, USA). The recombinant was named pEGFP-BNIPL-2. The transfection of plasmid DNAs into MHCC97-L cells was performed using Lipofectamine (Invitrogen, Carlsbad, CA, USA). Briefly, $1 \times 10^{5}$ cells were seeded per well into 6-well plates (Corning Inc., Corning, NY, USA). The cells were transfected using $1 \mu \mathrm{g}$ DNA mixed with $7 \mu 1$ Lipofectamine in $1 \mathrm{ml}$ DMEM without serum and antibiotics. The cells were incubated for $6 \mathrm{~h}$, and then $1 \mathrm{ml}$ DMEM containing 20\% serum was added into each well. The cells were cultured and screened in the medium containing $10 \%$ serum and $2 \mathrm{mg} / \mathrm{ml} \mathrm{G} 418$ (Invitrogen) for at least two weeks till the non-transfected MHCC97-L cells cultured in the controlled wells were all killed. A colony transfected stably with pEGFP-BNIPL-2 was selected, and this was designated as the MHCC97-L BNIPL-2 cells. The stable transfectants of MHCC97-L transfected with the pEGFP-N1 vector control were called MHCC97-L VC.

Immunoblotting. Immunoblot experiments were performed according to standard procedures. Briefly, the cells (MHCC97L BNIPL-2 or MHCC97-L VC cells) were harvested in lysis buffer [T-PER ${ }^{\mathrm{TM}}$ tissue protein extraction reagent (Pierce Chemical Company, Rockford, IL, USA) containing 1\% proteinase inhibitor cocktail] and clarified by centrifugation at $10,000 \mathrm{x} \mathrm{g}$. For protein extraction from the tissues, the samples were snap-frozen and immediately homogenized in lysis buffer. Equal amounts of protein $(20 \mu \mathrm{g} /$ lane $)$ were electrophoresed on $10 \%$ SDS-PAGE and blotted to the PROTRAN nitrocellulose transfer membrane. The rabbit polyclonal BNIPL-2 antibody was made by our laboratory (5). Monoclonal anti- $\beta$-actin and the anti-CD44 antibodies were from Sigma-Aldrich (St. Louis, MO, USA). Secondary anti-mouse or anti-rabbit antibodies coupled to horseradish peroxidase were from Santa Cruz Biotechnology (Santa Cruz, CA, USA). The immunocomplexes were visualized using the SuperSignal west femto maximum sensitivity substrate (Pierce Chemical Company).

Cell invasion assays. The invasive potential was measured by an in vitro Boyden chamber assay (8) with some modifications. Briefly, the cells were treated with trypsin-EDTA and counted. MHCC97-L BNIPL-2 or MHCC97-L VC cells $\left(4 \times 10^{4}\right)$ in 0.1 $\mathrm{ml}$ serum-free DMEM were added to the well of the $8-\mu \mathrm{m}$ pore membrane Boyden chamber (Costar, Cambridge, MA, USA) coated with Matrigel. The lower chambers contained $10 \%$ FBS in DMEM, serving as a chemoattractant. The cells were allowed to invade for $24 \mathrm{~h}$. The cells that had not penetrated the filters were removed by scrubbing with cotton swabs. The chambers were fixed for $20 \mathrm{~min}$ at room temperature with $4 \%$ formaldehyde in PBS, stained in $0.5 \%$ crystal violet for $20 \mathrm{~min}$, and rinsed in water. The cells that migrated to the lower surface of the filter were considered to have invaded the matrix and were counted with a light microscopy.

Wound healing assays. Wound healing assay was performed to study the effect of BNIPL-2 on cell motility, following the previous report with slight modifications (9). In this assay, MHCC97-L BNIPL-2 or MHCC97-L VC cells were grown to confluence in 60-mm dishes, and then the 'wound' was created by stripping a sharply defined channel using a razorblade. To prevent growth during migration, the cells were pretreated with $2 \mathrm{mM}$ thymine (Sigma-Aldrich) for $24 \mathrm{~h}$. After band-stripping, the cells were allowed to migrate into the wound for $24 \mathrm{~h}$, fixed with $4 \%$ formaldehyde in PBS for $20 \mathrm{~min}$, and then stained with crystal violet. The number of individual cells in the wound was quantitated as the average from six random fields under the microscope.

Xenograft model. A metastasis model was made on mice as described previously (10). Cells (5x106) (MHCC97-L BNIPL-2 or MHCC97-L VC cells) were subcutaneously injected into the right upper flank regions of experimental mice (4-6 weeks of age, male, BalB/c nu $/ \mathrm{nu}^{+}$, from the Shanghai Cancer Institute). The mice were housed in laminar flow cabinets under specific pathogen-free conditions. Once the subcutaneous tumor reached $1-1.5 \mathrm{~cm}$ in diameter, it was removed and cut into pieces of about $1 \times 1 \times 1 \mathrm{~mm}$ which were implanted into the liver of each of the 8 nude mice. After 35 days, the animals were sacrificed and autopsied. The mouse whose small tumor colonies around the tumor nodule derived from the implanted tumor tissue at the implantation site were visible to the naked eye, was considered as intrahepatic metastasis. The lungs were sampled for histopathological studies. The mouse whose metastatic HCC cells were found on any slide of the lung sections, was considered as lung metastasis. The animals were treated according to the guidelines established by the American Association for Laboratory Animal Care (AALAC).

Production of GST-PAK-CRIB domain (PAK-CD) fusion proteins. The cloning, expression and purification of the glutathione-S-transferase (GST)-PAK-CD fusion protein (encompassing amino acids 56-272 from PAK1B), containing the $\mathrm{Cdc} 42$ binding region from human PAK1B, have been described previously (11). In brief, Escherichia coli Top10F' cells were transformed with the GST-PAK-CD construct. The expression of the recombinant protein was induced by the addition of $0.1 \mathrm{mM}$ isopropylthiogalactoside. The cells were harvested, resuspended in PBS containing 1\% proteinase inhibitor cocktail, and then sonicated. The cell lysates were centrifuged and the supernatant was incubated with glutathione-coupled Sepharose 4B beads (Amersham Pharmacia Biotech, Piscataway, NJ, USA) for $30 \mathrm{~min}$ at $4^{\circ} \mathrm{C}$. The protein bound to the beads was washed three times in PBS and the amount of fusion protein was estimated using Coomassiestained SDS gel.

Cdc42 activation assays. Cells $\left(5 \times 10^{6}\right)$ were lysed in lysis buffer (T-PER ${ }^{\mathrm{TM}}$ tissue protein extraction reagent containing $1 \%$ proteinase inhibitor cocktail), clarified by centrifugation, and incubated with $200 \mu \mathrm{g}$ bacterially produced GST-PAK$\mathrm{CD}$ fusion protein for $60 \mathrm{~min}$. The proteins bound to the fusion protein were precipitated by the use of glutathioneSepharose beads and separated by SDS-PAGE, followed by immunoblotting with specific antibodies for $\mathrm{Cdc} 42$.

Cell morphological observation. MHCC97-L cells $\left(5 \times 10^{4}\right)$ were transiently transfected for $24 \mathrm{~h}$ with the pEGFP-N1 or pEGFP-BNIPL-2 plasmid, and then they were loaded onto 


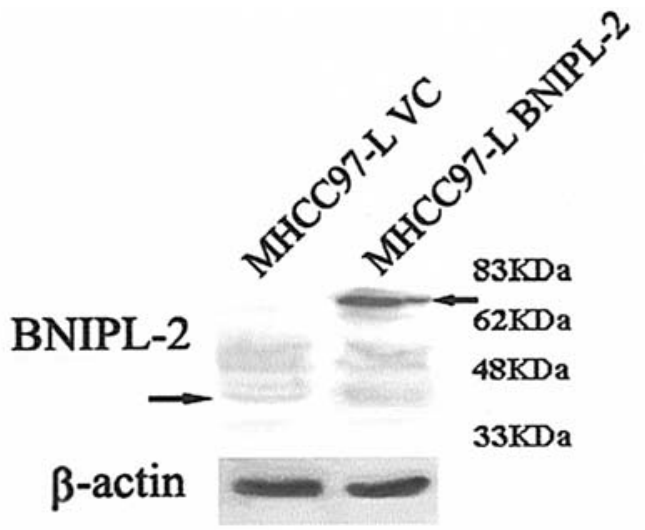

Figure 1. Immunoblot analysis of BNIPL-2 expression. Equal amounts of total cell protein were analyzed using the BNIPL-2 antibody. Positions of the molecular weight markers are indicated on the right. $\beta$-actin is shown as the loading control.

glass coverslips. After incubation at $37^{\circ} \mathrm{C} / 5 \% \mathrm{CO}_{2}$, cell morphology was viewed with a microscope by direct fluorescence detection and representative pictures were taken.

Statistical analysis. All the experiments were performed in triplicates as indicated. Fisher's exact test and the Student's t-test were used, respectively, for comparisons of the enumeration data (number of mice with metastases) and other measurement data. $\mathrm{P}<0.05$ was considered as statistically significant.

\section{Results}

Overexpression of BNIPL-2 in MHCC97-L cells. MHCC97-L cells were transfected with the pEGFP-BNIPL-2 or pEGFPN1 vector. The expression of BNIPL-2 in the transfectants was analyzed by immunoblotting using the BNIPL-2 antibody (Fig. 1). In the MHCC97-L VC cells, the band of endogenous BNIPL-2 expression migrated at the expected position ( $\sim 3 \mathrm{kDa})$, which was almost invisible. The major band of remarkably overexpressed BNIPL-2 migrated at $\sim 70 \mathrm{kDa}$ in the MHCC97-L BNIPL-2 cells.

Cell invasion and migration of MHCC97-L BNIPL-2 cells. In the cell invasion assays, the MHCC97-L cells were found to exhibit a pronounced cell invasion. The MHCC97-L BNIPL-2 cells that migrated to the lower surface of the filter were five times more than the MHCC97-L VC cells (Fig. 2A). Wound healing assay was then used as a measure of the rate of cell migration or movement (12). The data indicate that the MHCC97-L BNIPL-2 cells migrating into the wound were about seven times as many as the MHCC97-L VC cells (Fig. 2B). These results demonstrate that the overexpression of BNIPL-2 in MHCC97-L cells promotes cell invasion and migration in vitro $(\mathrm{P}<0.05)$.

BNIPL-2 promotes the metastasis of HCC cells in vivo. The xenografts were implanted into the livers of two nude mice groups. When the animals were sacrificed and autopsied, we found that the BNIPL-2 protein was still overexpressed in the xenografts of the MHCC97-L BNIPL-2 group (Fig. 3A). Four
$\mathbf{A}$

A MHCC97-L VC

MHCC97-L BNIPL-2
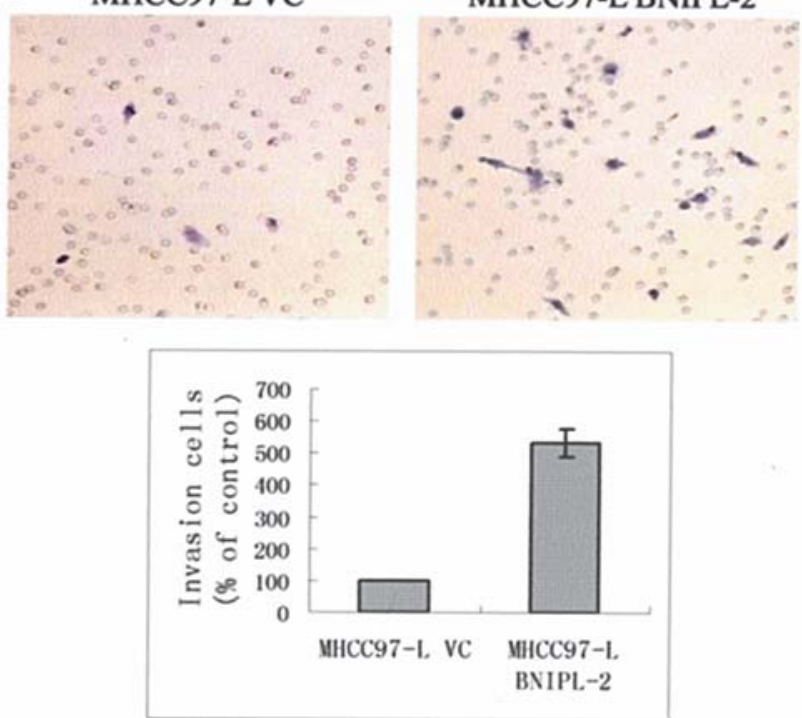

B

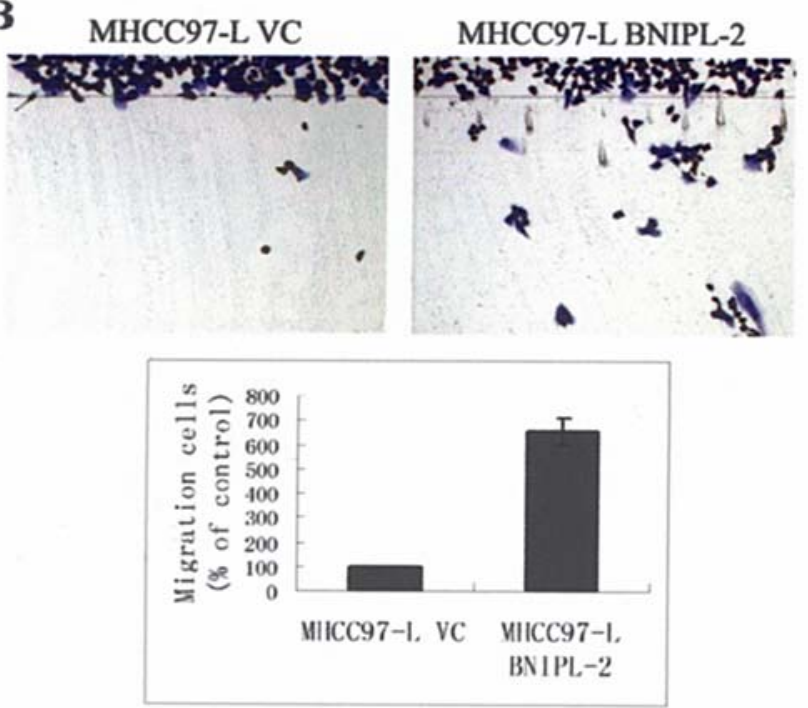

Figure 2. BNIPL-2 promoted invasion and migration in vitro. The results are the average of three independent experiments with five fields counted per experiment. Bars represent the SE. Data are expressed as the means \pm SE from three replicate experiments $(\mathrm{P}<0.05)$. (A) Matrigel invasion assay indicated an increase in cell invasion (x100 magnification). The fields show the attached cells that successfully reached out and invaded the other side of the Matrigel membrane. Cells passing through the Matrigel membrane were found to increase significantly when BNIPL-2 was overexpressed. (B) The wound-healing assay shows the effects of BNIPL-2 on the motility of human hepatocellular carcinoma cells with low metastatic potential (MHCC97-L) (x100 magnification). A significant acceleration in cell motility was revealed in the MHCC97-L BNIPL-2 cells compared with the control.

of 7 mice in the MHCC97-L BNIPL-2 group showed intrahepatic metastasis (Fig. 3B). The metastatic ratio of intrahepatic metastasis was obviously increased compared with the MHCC97-L VC group (0/7,0\%), as shown in Table I. Furthermore, pulmonary metastasis was observed in the lung tissue sections (Fig. 3C) in the MHCC97-L BNIPL-2 group $(7 / 7,100 \%)$, much more than the ratio of pulmonary metastasis in the MHCC97-L VC group (2/7, 28.6\%, Table I). These results indicate that the overexpression of BNIPL-2 enhanced the metastatic ability of the HCC cells in vivo. 


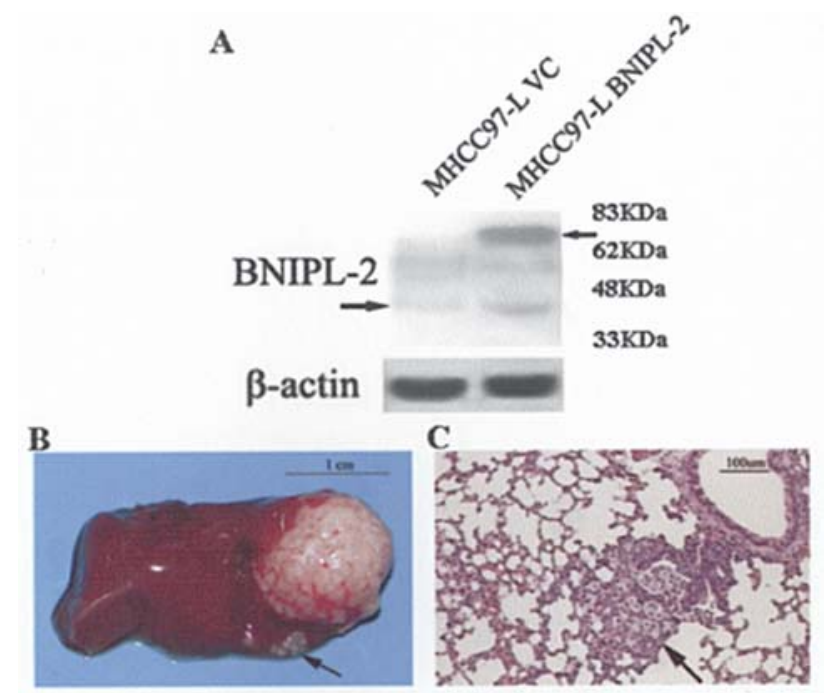

Figure 3. BNIPL-2 enhanced cancer metastasis in vivo. (A) BNIPL-2 expression in the xenografts. Lysates from the xenografts were immunoblotted for detection of the BNIPL-2 expression. Positions of the molecular weight markers are indicated on the right. $\beta$-actin is shown as the loading control. (B) Intrahepatic metastasis in the xenografts. The black arrow represents the metastatic nodules. (C) Pulmonary metastasis in the xenograft models. The black arrow represents the metastatic human HCC cells in the lung tissues of the mice. H\&E staining.

Table I. Comparison of the metastatic ratio between the MHCC97-L VC and MHCC97-L BNIPL-2 group.

\begin{tabular}{llll}
\hline & $\begin{array}{c}\text { MHCC97-L } \\
\text { VC }\end{array}$ & $\begin{array}{c}\text { MHCC97-L } \\
\text { BNIPL-2 }\end{array}$ & P-value \\
\hline Intrahepatic & $0 / 7,0 \%$ & $4 / 7,57.1 \%$ & 0.035 \\
Pulmonary & $2 / 7,28.6 \%$ & $7 / 7,100 \%$ & 0.010
\end{tabular}

$\mathrm{P}$ indicates the possibility using Fisher's exact test

Cdc42 activition and filopodia formation. The GST-PAK-CD protein was used to pull down the activated $\mathrm{Cdc} 42, \mathrm{Cdc} 42-$ GTP, from the extracts of the cells. As shown in Fig. 4A, the basal activity of Cdc42 was low in the MHCC97-L VC cells; and the MHCC97-L BNIPL-2 cells had an obvious increase in the level of Cdc42-GTP.

With direct fluorescence microscopy, we found that the MHCC97-L cells transiently transfected with the pEGFP-N1 plasmid were mostly round in shape and $<5 \%$ of them had some background with irregular shapes including filopodia. However, when the plasmid DNAs of pEGFP-BNIPL-2 were transiently transfected into the MHCC97-L cells, the cells were more irregular in shape. BNIPL-2 induced filopodia formation in almost $50 \%$ of the transfected cells (Fig. 4B). The data reveal that BNIPL-2 caused the increase in Cdc42 activity and the formation of filopodia, which led to cell migration and cancer metastasis.

Upregulation of CD44 in MHCC97-L BNIPL-2 cells. Using Western blotting, we examined the expression of the cell

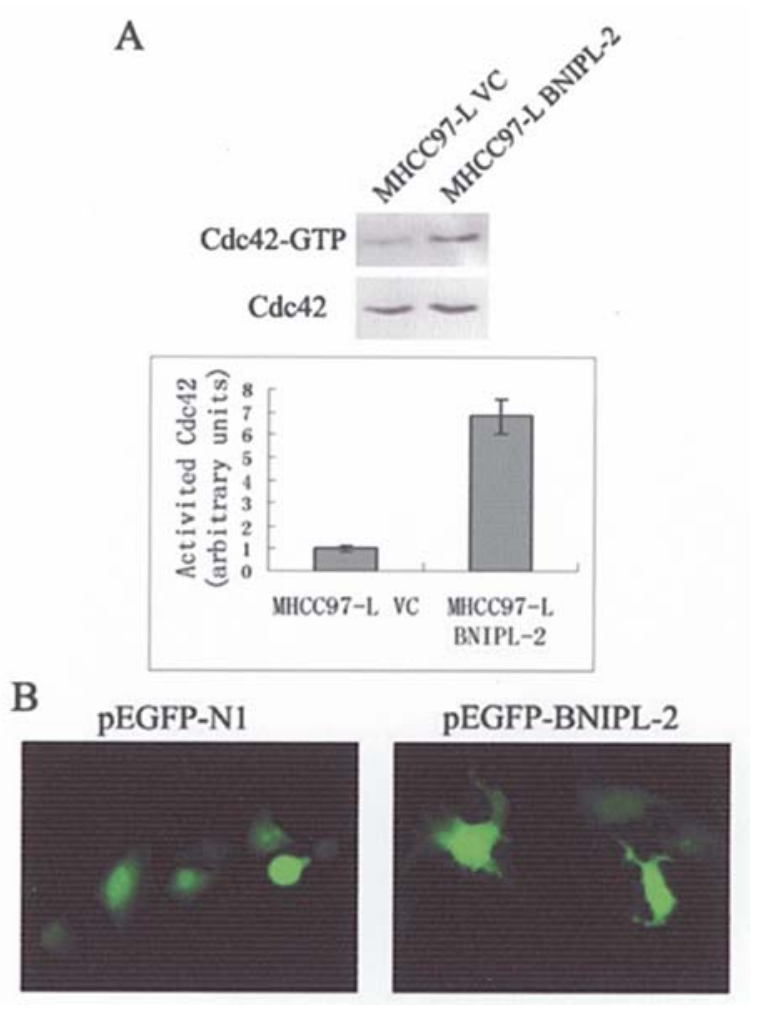

Figure 4. Cdc42 GTPase activation and filopodia formation. (A) The activation of $\mathrm{Cdc} 42$. The top panel shows the active $\mathrm{Cdc} 42$ in the GTPbound form; the lower panel indicates the total amount of $\mathrm{Cdc} 42$ in $10 \mu \mathrm{g}$ total cell extract. The intensity of the bands corresponding to Cdc42-GTP was quantified according to the amount of total $\mathrm{Cdc} 42$. The results are shown by the means \pm SE of three independent experiments $(\mathrm{P}<0.05)$. (B) BNIPL-2 induced filopodia formation. The MHCC97-L cells transiently transfected for $24 \mathrm{~h}$ with the GFP expression plasmids pEGFP-BNIPL-2 or the control

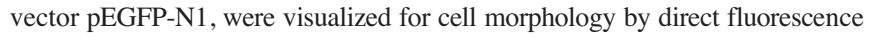
detection.

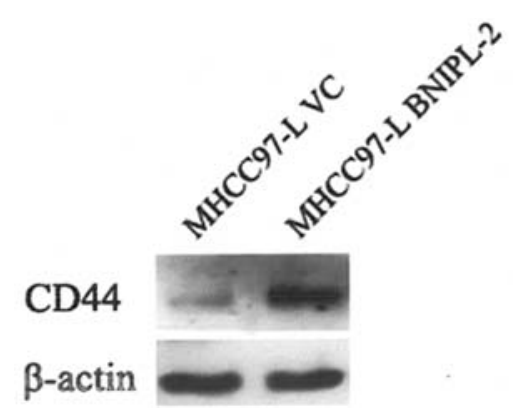

Figure 5. The effects of BNIPL-2 on CD44 expression in cancer cells. CD44 was highly expressed in the MHCC97-L BNIPL-2 cells. Lysates from the cell lines were immunoblotted in order to examine the expression of CD44. B-actin is shown as the loading control.

surface adhesion molecule, CD44, which was involved in cell migration and invasion. Our results show that the overexpression of BNIPL-2 in the MHCC97-L cells led to the upregulation of the protein expression of CD44 (Fig. 5).

\section{Discussion}

HCC is one of the most common and aggressive human malignancies (13). Primary HCC can be partly treated with 
either surgery or chemotherapy. However, a major complication of such treatment regimens results from the development of metastases, the distant tumor colonies derived from the spread of cancer cells from a primary tumor through the blood or lymphatic system (14). Despite remarkable advances in diagnostic and therapeutic techniques for cancer, the prognosis of HCC patients remains poor due to metastasis.

The overexpression or deregulation of the GTPase or some element of the Rho pathway has been reported in many human cancers (15). Rho-family GTPases are a multimember family of proteins involved in diverse cellular functions that are related to cell growth, development, apoptosis, tumorigenesis, and metastasis (16). Our previous assays of proteinprotein interactions have shown that BNIPL-2 interacted with Cdc42GAP through the $\mathrm{BCH}$ domain locateed at the $\mathrm{C}$ terminus and suppressed the Cdc42GAP activity towards Cdc42 by adding BNIPL-2 into the in vitro GAP activity assay system (5). However, it is still unclear whether BNIPL-2 is involved in the process of cancer metastasis via the $\mathrm{Cdc} 42$ pathway.

Since the signal pathways triggered by $\mathrm{Cdc} 42$ modulate distinct cytoskeletal rearrangement and the formation of filopodia for cell motility, we wanted to elucidate whether BNIPL-2 affects the morphology and migration potentials of the target cells via the Cdc42 pathway. In this report, our data indicate that BNIPL-2 not only enhanced the migration and invasion of cancer cells in vitro (Fig. 2), but also promoted cancer metastasis in vivo (Fig. 3, Table I), which was consistent with the result that BNIPL-2 was involved in the activition of the small GTPase, $\mathrm{Cdc} 42$, and the promotion in filopodia formation (Fig. 4).

We also selected another stable cell line expressing the BNIPL-2 protein in the same conditions and repeated the metastatic assays in vitro and in vivo. Enhanced cell migration and cancer metastasis were also observed, although the level of BNIPL-2 overexpression in the second stable cells was almost $20 \%$ as much as in the MHCC97-L BNIPL-2 cells (data not shown).

CD44 is a transmembrane receptor for the extracellular matrix (ECM) components including hyaluronic acid (HA) (17) and is implicated in a wide variety of adhesion-dependent cellular processes including lymphocyte homing (18), cell migration $(19,20)$, and tumor cell metastasis and invasion (21-23). The activation of the Rho family of small $\mathrm{G}$ proteins, Cdc42, is responsible for the Ha-RasVal-12-induced CD44 cleavage $(24,25)$. CD44 expressed in cancer cells is proteolytically cleaved through membrane-anchored metalloproteases and CD44 cleavage plays a critical role in cancer cell migration $(25,26)$. Therefore, we performed Western blot analysis with the antibody against CD44 and found that CD44 expression waas upregulated when BNIPL-2 was overexpressed in MHCC97-L cells (Fig. 5), which facilitated cancer cell migration and invasion. However, the molecular pathway of CD44 expression upregulated by BNIPL-2 remains to be elucidated.

As cancer metastasis is a complicated process, many molecular characteristics are still unknown. In the present study, we firstly provided evidence that BNIPL-2 increases the invasion of cancer cells in vitro and promotes the metastasis of human HCC in vivo. Furthermore, it is the potential molecular mechanism that BNIPL-2 enhances cancer metastasis via maintaining the high level of the active Cdc42 form, Cdc42-GTP, and upregulating the expression of CD44. These results link BNIPL-2 with cancer metastasis and allow us to further analyze its signaling pathway involved in the invasion and metastasis of cancer.

\section{Acknowledgements}

We thank Yuan Han, Mingxia Yan, Fengrui Zhang, Chao Ge, Shumin Qu, and Wei Zhang for their skillful technical support. This study was supported by a grant from the National Key Basic Research Program (973) of China (No. 2002CB513104), a grant from the Program of Shanghai Subject Chief Scientist (05XD14013), and a grant from the NSFC-RGC Joint Fund 30218006.

\section{References}

1. Pantel K and Brakenhoff RH: Dissecting the metastatic cascade. Nat Rev Cancer 4: 448-456, 2004.

2. Couzin J: Medicine. Tracing the steps of metastasis, cancer's menacing ballet. Science 299: 1002-1006, 2003.

3. Friedl $\mathrm{P}$ and Wolf $\mathrm{K}$ : Tumour-cell invasion and migration: diversity and escape mechanisms. Nat Rev Cancer 3: 362-374, 2003.

4. Etienne-Manneville S and Hall A: Rho GTPases in cell biology. Nature 420: 629-635, 2002.

5. Qin W, Hu J, Guo M, et al: BNIPL-2, a novel homologue of BNIP-2, interacts with Bcl-2 and Cdc42GAP in apoptosis. Biochem Biophys Res Commun 308: 379-385, 2003.

6. Shang X, Zhou YT and Low BC: Concerted regulation of cell dynamics by BNIP-2 and Cdc42GAP homology/Sec14p-like, proline-rich, and GTPase-activating protein domains of a novel Rho GTPase-activating protein, BPGAP1. J Biol Chem 278: 45903-45914, 2003.

7. Tang ZY, Ye SL, Liu YK, et al: A decade's studies on metastasis of hepatocellular carcinoma. J Cancer Res Clin Oncol 130: 187-196, 2004.

8. Albini A, Iwamoto $\mathrm{Y}, \mathrm{K}$ leinman $\mathrm{HK}$, et al: A rapid in vitro assay for quantitating the invasive potential of tumor cells. Cancer Res 47: 3239-3245, 1987.

9. Andre F, Rigot V, Remacle-Bonnet M, Luis J, Pommier G and Marvaldi J: Protein kinases C-gamma and -delta are involved in insulin-like growth factor I-induced migration of colonic epithelial cells. Gastroenterology 116: 64-77, 1999

10. Lu X, Qin W, Li J, et al: The growth and metastasis of human hepatocellular carcinoma xenografts are inhibited by small interfering RNA targeting to the subunit ATP6L of proton pump. Cancer Res 65: 6843-6849, 2005.

11. Sander EE, ten Klooster JP, van Delft S, van der Kammen RA and Collard JG: Rac downregulates Rho activity: reciprocal balance between both GTPases determines cellular morphology and migratory behavior. J Cell Biol 147: 1009-1022, 1999.

12. Fenteany G, Janmey PA and Stossel TP: Signaling pathways and cell mechanics involved in wound closure by epithelial cell sheets. Curr Biol 10: 831-838, 2000.

13. Pisani P, Parkin DM, Bray F and Ferlay J: Estimates of the worldwide mortality from 25 cancers in 1990. Int J Cancer 83: 18-29, 1999

14. Ye QH, Qin LX, Forgues M, et al: Predicting hepatitis B viruspositive metastatic hepatocellular carcinomas using gene expression profiling and supervised machine learning. Nat Med 9: 416-423, 2003.

15. Sahai E and Marshall CJ: RHO-GTPases and cancer. Nat Rev Cancer 2: 133-142, 2002.

16. Schmitz AA, Govek EE, Bottner B and Van Aelst L: Rho GTPases: signaling, migration, and invasion. Exp Cell Res 261: $1-12,2000$.

17. Aruffo A, Stamenkovic I, Melnick M, Underhill CB and Seed B: CD44 is the principal cell surface receptor for hyaluronate. Cell 61: 1303-1313, 1990.

18. Goldstein LA, Zhou DF, Picker LJ, et al: A human lymphocyte homing receptor, the hermes antigen, is related to cartilage proteoglycan core and link proteins. Cell 56: 1063-1072, 1989. 
19. Thomas L, Byers HR, Vink J and Stamenkovic I: CD44H regulates tumor cell migration on hyaluronate-coated substrate. J Cell Biol 118: 971-977, 1992.

20. Svee K, White J, Vaillant P, et al: Acute lung injury fibroblast migration and invasion of a fibrin matrix is mediated by CD44. J Clin Invest 98: 1713-1727, 1996.

21. Yu Q and Stamenkovic I: Localization of matrix metalloproteinase 9 to the cell surface provides a mechanism for CD44mediated tumor invasion. Genes Dev 13: 35-48, 1999.

22. Takahashi K, Eto $\mathrm{H}$ and Tanabe KK: Involvement of CD44 in matrix metalloproteinase-2 regulation in human melanoma cells. Int J Cancer 80: 387-395, 1999.

23. Gunthert U, Hofmann M, Rudy W, et al: A new variant of glycoprotein CD44 confers metastatic potential to rat carcinoma cells. Cell 65: 13-24, 1991.
24. Kawano Y, Okamoto I, Murakami D, et al: Ras oncoprotein induces $\mathrm{CD} 44$ cleavage through phosphoinositide 3-OH kinase and the rho family of small $\mathrm{G}$ proteins. J Biol Chem 275: 29628-29635, 2000.

25. Okamoto I, Kawano Y, Matsumoto M, et al: Regulated CD44 cleavage under the control of protein kinase $\mathrm{C}$, calcium influx, and the Rho family of small G proteins. J Biol Chem 274: 25525-25534, 1999.

26. Okamoto I, Kawano Y, Tsuiki H, et al: CD44 cleavage induced by a membrane-associated metalloprotease plays a critical role in tumor cell migration. Oncogene 18: 1435-1446, 1999. 